\title{
Asymptomatic cerebellar medulloblastoma unmasked by minor head injury
}

\author{
J J McInerney, A J Colquhoun, G G Bodiwala
}

Patients who reattend the accident and emergency (A\&E) department after a minor head with persistent symptoms of headache, dizziness, and fatigability are diagnosed commonly as suffering from postconcussional syndrome. ${ }^{1}$ Clinicians should be aware, however, that minor head injury may unmask previously asymptomatic neurological diseases, including tumours, berry aneurysms, arteriovenous malformations, and cerebral abscesses. ${ }^{2}$ We report such a case of asymptomatic cerebellar medulloblastoma presenting after minor head injury.

A 17 year old man presented five days after receiving a forceful blow to the head from a football, which resulted in him feeling dazed momentarily. He had experienced no neurological symptoms before this event but described a history of headache and dizziness persisting from the time of injury. Initial examination revealed a Glasgow coma score of 15 . Full neurological examination revealed no abnormality and he was diagnosed as having postconcussional syndrome and discharged with head injury advice. He returned four days later with symptoms of increasing headache, vomiting, dizziness, and drowsiness. $\mathrm{He}$ was admitted to the A\&E short stay ward where further examination revealed a Glasgow coma score of 15 . Repeat neurological examination revealed bilateral papilloedema and up-beat nystagmus. Computed tomography of the brain with contrast (fig 1) showed a hyperattenuating mass in the right cerebellar hemisphere with distortion of the fourth ventricle, and triventricular hydrocephalus. $\mathrm{He}$ was transferred to the regional neurosurgical centre

Accident and Emergency

Department, Leicester

Royal Infirmary,

Infirmary Close,

Leicester LE1 5WW

J J McInerney

A J Colquhoun

G G Bodiwala

Correspondence to: Dr McInerney and underwent surgical excision of a large multilobular tumour from the superior aspect of the cerebellar vermis. There were no complications and his hydrocephalus settled rapidly. Subsequent histology showed the mass to be a cerebellar medulloblastoma or primitive neuroectodermal tumour.

The mechanism whereby asymptomatic brain tumours are unmasked by minor trauma

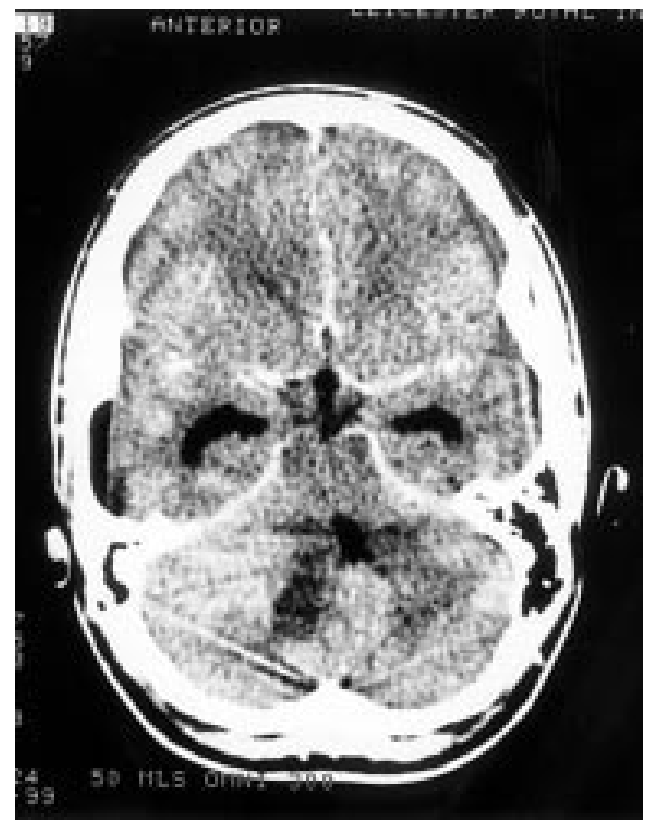

Figure 1 Computed tomogram of the brain.

is unclear. It is feasible that direct trauma to the tumour may result in oedema and hydrocephalus leading to symptoms resembling the postconcussional syndrome. Although cerebellar medulloblastoma is rare in adults, ${ }^{3}$ postconcussional syndrome is common. ${ }^{1}$ However patients who reattend after minor head trauma have been shown to represent a high risk group. ${ }^{4}$ Accordingly a short period of observation should always be considered and early computed tomography undertaken in those whose symptoms persist.

\footnotetext{
1 Lowdon IM, Briggs M, Cockin J. Post-concussional symptoms following minor head injury. Injury 1989;20:193-4

2 Wallis WE, Wilson J. Head injury unmasking other brain diseases. Acta Neurol Suppl 1983;32:125-7.

3 Tekkok IH, Suzer T, Ozgen T, et al. Cerebellar medulloblastoma in adults. Neurosurg Rev 1991;14:135-40.

4 Voss M, Knottenbelt JD, Peden MM. Patients who reattend after head injury: a high risk group. BMF 1995;311:1395-8.
} 